\title{
Genetic Liability to Cannabis Use Disorder and COVID-19 Hospitalization
}

\author{
Alexander S. Hatoum ${ }^{1}$, Claire L. Morrison ${ }^{2}$, Evan A. Winiger ${ }^{2}$, Emma C. Johnson ${ }^{1}$, Arpana \\ Agrawal $^{*}$, Ryan Bogdan ${ }^{3 *}$
}

${ }^{1}$ Washington University School of Medicine, Department of Psychiatry

${ }^{2}$ Institute for Behavioral Genetics, University of Colorado Boulder

${ }^{3}$ Washington University in St. Louis, Department of Psychological \& Brain Sciences

\section{Email: ashatoum@wustl.edu}

ORCID ID: https://orcid.org/0000-0002-8002-7267

\section{Author Contributions:}

ASH, ECJ, AA, and RB developed the research questions. ASH conducted analyses, with help from CLM and EAW. ASH drafted the manuscript. ECJ, AA, and RB provided critical revision of the manuscript for important intellectual content. ASH had full access to all data in the study and take responsibility for the integrity of the data and accuracy of the data analyses.

\section{Competing Interest Statement: We have no financial interests to disclose.}

Classification: Major: Social and Biological Sciences. Minor: Genetics, Medical Sciences, Psychological and Cognitive Sciences

Keywords: Cannabis Use Disorder, Genome-wide association statistics, COVID-19, genetic correlations, quasi-experimental design, tobacco use.

\section{Acknowledgments}

ASH receives support from DA007261-17. CLM receives support from MH016880. EAW receives support from HD007289-30. ECJ receives support from AA027435-02. AA receives support from MH109532 and K02DA032573 Dr. Bogdan (AG052564, AA027827, DA046224). We also would like to acknowledge the COVID Host Genomics Consortium for their open data policies. All data are available through the PGC, GSCAN and COVID-HG consortiums. Conflict of interest disclosures: No disclosures were reported.

\section{This file includes:}

Main text length: 1040 words

Manuscript length: 1460 words

Literature Citations: 5

GWAS data Citations: 6

Method Citations: 4

Figure: 1

Table: 1 
medRxiv preprint doi: https://doi.org/10.1101/2020.11.15.20229971; this version posted November 18, 2020. The copyright holder for this preprint (which was not certified by peer review) is the author/funder, who has granted medRxiv a license to display the preprint in perpetuity.

It is made available under a CC-BY-NC-ND 4.0 International license .

\begin{abstract}
Behavioral and life style factors plausibly play a role in likelihood of being hospitalized for COVID19. Genetic vulnerability to hospitalization after SARS-CoV2 infection may partially relate to comorbid behavioral risk factors, especially the use of combustible psychoactive substances. Paralleling the COVID-19 crisis has been increasingly permissive laws for recreational cannabis use. Cannabis Use Disorder (CUD) is a psychiatric disorder that is heritable and genetically correlated with respiratory disease, independent of tobacco smoking. By leveraging genome-wide association summary statistics of CUD and COVID-19, we find that at least $1 / 3^{\text {rd }}$ of the genetic vulnerability to COVID-19 overlaps with genomic liability to CUD ( $r g=.34, p=0.0003)$. Genetic causality as a potential mechanism of risk could not be excluded. The association between CUD and COVID-19 remained when accounting for genetics of trying marijuana, tobacco smoking (ever smoking regularly, cigarettes per day, smoking cessation, age of smoking initiation), BMI, fasting glucose, forced expiration volume, education attainment, and Townsend deprivation index. Heavy problematic cannabis use may increase chances of hospitalization due to COVID-19 respiratory complications. Curbing excessive cannabis use may be an essential strategy in COVID-19 mitigation.
\end{abstract}




\section{Main Text}

Introduction. Paralleling the Coronavirus Disease 2019 (COVID-19) pandemic has been an increase in substance use (1) and a continuation of increasingly permissive laws surrounding cannabis. On November $3^{\text {rd }}, 2020$, American voters in 4 U.S. states (Arizona, Montana, New Jersey, South Dakota) voted to join 11 that have already legalized recreational cannabis use. Legalization is associated with increased use and $20 \%$ of individuals who have tried cannabis develop cannabis use disorder (CUD) (2), a moderately heritable (50-60\%) psychiatric syndrome that shares genetic risk with respiratory disease (3). As the heterogeneous presentation of COVID-19 is partially attributable to host genomic background and respiratory symptoms are the primary reason for hospitalization and death (4), genomic liability to CUD may contribute to severe COVID-19 presentation.

Results. We leveraged genome-wide association study (GWAS) summary statistics to estimate whether genetic liability to CUD ( $\mathrm{n}$ case $=14,080$, $n$ control $=343,726$ ) (3) may plausibly influence COVID-19 hospitalization ( $\mathrm{n}$ case $=6,492$, $\mathrm{n}$ population control=1,012,809) (4). First, Linkage Disequilibrium Score Regression (5) analyses revealed that genetic liability for CUD and COVID19 hospitalization are correlated $\left(S N P-r_{g}=0.34, p=0.0003\right.$, and remained significant after Bonferroni correction for pairwise genetic correlations across all traits; Figure 1). Second, genetic liability to tobacco smoking phenotypes was also shared with COVID-19 hospitalization (Figure 1). A series of genomic structural equation models (gSEM) (6) revealed an independent genomic association between CUD and COVID-19 hospitalization (Table 1) when accounting for genomic liability to tobacco phenotypes (i.e., cigarettes per day, age of becoming a regular smoker, ever being a regular smoker, smoking cessation(7)), lifetime cannabis use(8), cardiometabolic traits (i.e., BMI (9), fasting glucose (10)), respiration (i.e., 1-second forced expiratory volume (11)) and socioeconomic status (i.e., educational attainment (12), Townsend deprivation index (11)). The only tobacco phenotype that retained a significant independent association in these models was smoking cessation, which was protective (Table 1). Third, and finally, latent causal variable (13) (LCV) analyses provided evidence that liability to CUD may be genetically causal for COVID-19 hospitalization (genetic causality proportion estimate $=0.63(.21), \mathrm{p}=4.0 \mathrm{e}-6)$.

Discussion. Our findings suggest that genetic liability to CUD is correlated with, and might increase risk for, COVID-19 hospitalization. The genetic correlation between CUD and COVID-19 hospitalization was independent of potential confounding variables (e.g., other substance inhalation phenotypes, cardio-metabolic traits, respiration, socioeconomic status indicators). On the other hand, genetic liability to lifetime cannabis ever-use showed a non-significant protective effect for COVID-19 hospitalization; divergent directionality of cannabis use and CUD has been observed for other phenotypes (e.g., BMI) (3). While highly speculative, it is possible that heavy and/or problematic cannabis use associated with CUD may increase COVID-19 severity, while any lifetime use may not be a marker of genetic susceptibility. Other putative mechanisms warrant attention in future research (e.g., potential shared effects on ACE2, inflammation). The use of population controls (i.e., individuals who may or may not have had COVID-19) in the COVID-19 hospitalization GWAS may have impacted association estimate precision and the predominant composition of European ancestry in constituent GWASs may limit generalizability to other ancestral populations. Finally, LCV models were unstable when only SNPs with MAF $>0.05$ were included and as such await replication (simulations suggest this may be attributable to reduced SNP density as opposed to MAF threshold; estimates of the GCP remained the same at 0.05 and were significant, but with wider standard errors). As the world prepares for surges in COVID-19, identifying putative risk factors associated with severe presentations may mitigate its 
worldwide impact. In contrast to anecdotal evidence and media reports (14) that cannabis may attenuate COVID-19, these data urge caution in heavy cannabis use during the COVID-19 pandemic.

Materials and Methods. The following genome-wide association study (GWAS) summary statistics were used in analyses: CUD ( $\mathrm{n}$ case $=14,080, \mathrm{n}$ control $=343,726)(3)$ COVID-19 hospitalization ( $\mathrm{n}$ case $=6,492$, $\mathrm{n}$ population control=1,012,809) (4), $\mathrm{BMI}(\mathrm{N}=795,640)(9)$, fasting glucose $(\mathrm{N}=58,074)(10)$, ever trying marijuana $(\mathrm{N}=184,765)$ (8), Forced Expiration Volume (11) (FEV; $\mathrm{N}=272,338)$, Townsend deprivation index (11) (TDI; $\mathrm{N}=336,798)$, education attainment (12) $(N=766,345)$, cigarettes per day $(N=263,954)$, ever a regular smoker $(n=632,802)$, smoking cessation ( $\mathrm{N}=312,821)$, and age of smoking initiation $(\mathrm{N}=262,990$; all smoking phenotypes are from the GSCAN consortium) (7). All GWAS summary statistics were generated from samples of genomically-confirmed European ancestry, with the exception of COVID-19 hospitalization which had $4.5 \%$ of the sample coming from other ethnic populations, and the remaining being of European descent.

LDSCORE: Linkage Disequilibrium Score Regression (LDSC) (5) was used to estimate genetic correlations between traits using single nucleotide polymorphisms (SNPs) with minor allele frequencies $(\mathrm{MAF})>0,01$, INFO > 0.90. Palindrome and multi-allelic single nucleotide polymorphisms (SNPs) as well as duplicate rs numbers and insertion/deletion polymorphisms were excluded before estimating genetic correlations. Single Nucleotide Polymorphisms (SNPs) with less than 1000 individuals were removed via the sample sizes of each GWAS (this was partially done to remove SNPs specific to anyone minority populations). LD score Beta weights and LD were pre-generated from 1000 Genomes European GWAS data included in the LDSC software download. LDSC regresses Chi-square statistics from the summary stats of GWAS on LD scores of the trait of interest (5). LD scores for each SNP are calculated via the sum of the variance explained by LD of that SNP with other SNPs (15). Genetic correlations were estimated using overlapping SNPs from filtered summary statistic files provided from GWAS and accounted for population stratification.

Modeling Genetic Covariances from LDSCORE: Using the pairwise genetic correlation matrix, Genomic Structural Equation Modeling (gSEM) allows for estimating multivariate models with the genetic correlation matrix as input data. In this case, we can parameterize a multiple regression, with CUD genetics predicting COVID-19 vulnerability above and beyond other variables of interest. Smoking phenotypes were tested in separate models to avoid multicollinearity among tobacco phenotypes.

Latent Causal Variable Analysis: LD scores can also be used to parameterize a quasiexperimental model. Building upon instrumental variable analyses and LDSR as applied to genomic data (often referred to as Mendelian Randomization), LCV models a latent "causal" variable which mediates the genetic correlation between two traits. This is quantified by the genetic causality proportion (GCP), an estimate of the degree to which each trait is correlated with the latent genetic variable, i.e., the extent to which each trait is potentially genetically causal (ranging from 0 reflecting no genetic causality to $|1|$ indicating full genetic causality). 


\section{References}

1. A. Farhoudian, et al., A global survey on changes in the supply, price and use of illicit drugs and alcohol, and related complications during the 2020 COVID-19 pandemic. medRxiv 13, 2020.07.16.20155341 (2020).

2. D. S. Hasin, US Epidemiology of Cannabis Use and Associated Problems. Neuropsychopharmacology 43, 195-212 (2018).

3. E. C. Johnson, et al., A large-scale genome-wide association study meta-analysis of cannabis use disorder. The Lancet Psychiatry 0 (2020).

4. , The COVID-19 Host Genetics Initiative, a global initiative to elucidate the role of host genetic factors in susceptibility and severity of the COVID-19 virus pandemic. Eur. J. Hum. Genet. 28, 715-718 (2020).

5. B. Bulik-Sullivan, et al., LD score regression distinguishes confounding from polygenicity in genome-wide association studies. Nat. Genet. (2015) https:/doi.org/10.1038/ng.3211.

6. A. D. Grotzinger, et al., Genomic structural equation modelling provides insights into the multivariate genetic architecture of complex traits. Nat. Hum. Behav. 3, 513-525 (2019).

7. M. Liu, et al., Association studies of up to 1.2 million individuals yield new insights into the genetic etiology of tobacco and alcohol use. Nat. Genet. 51, 237-244 (2019).

8. J. A. Pasman, et al., GWAS of lifetime cannabis use reveals new risk loci, genetic overlap with psychiatric traits, and a causal influence of schizophrenia. Nat. Neurosci. 21, 11611170 (2018).

9. L. Yengo, et al., Meta-analysis of genome-wide association studies for height and body mass index in 700 000 individuals of European ancestry. Hum. Mol. Genet. 27, 36413649 (2018).

10. A. K. Manning, et al., A genome-wide approach accounting for body mass index identifies genetic variants influencing fasting glycemic traits and insulin resistance. Nat. Genet. 44, 659-669 (2012).

11. T. H. team Liam Abbott, Sam Bryant, Claire Churchouse, Andrea Ganna, Daniel Howrigan, Duncan Palmer, Ben Neal, Raymond Walters, Caitlin Carey, Neale lab UKB GWAS Results. http://www.nealelab.is/uk-biobank.

12. J. J. Lee, et al., Gene discovery and polygenic prediction from a genome-wide association study of educational attainment in 1.1 million individuals. Nat. Genet. 50, 1112-1121 (2018).

13. L. J. O'Connor, A. L. Price, Distinguishing genetic correlation from causation across 52 diseases and complex traits. Nat. Genet. 50, 1728-1734 (2018).

14. , Cannabis Shows Potential To Help And Harm In Coronavirus Cases: Experts Explain Why (October 21, 2020).

15. B. Bulik-Sullivan, et al., An atlas of genetic correlations across human diseases and traits. Nat. Genet. (2015) https:/doi.org/10.1038/ng.3406. 
medRxiv preprint doi: https://doi.org/10.1101/2020.11.15.20229971; this version posted November 18, 2020. The copyright holder for this preprint (which was not certified by peer review) is the author/funder, who has granted medRxiv a license to display the preprint in perpetuity.

It is made available under a CC-BY-NC-ND 4.0 International license .

Figure 1. Genetic associations between Substance Use Phenotypes and COVID-19 Hospitalization

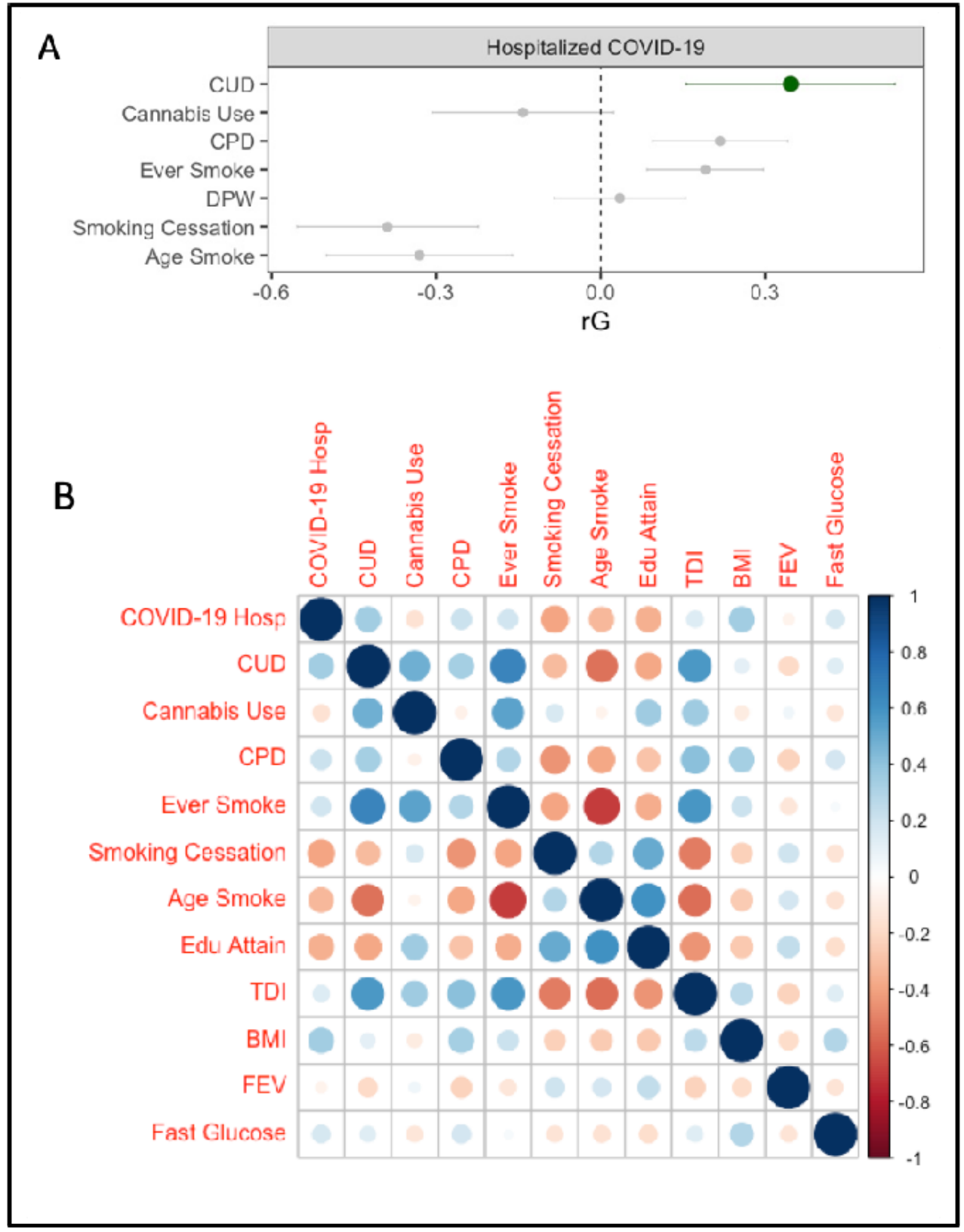

A) Genetic correlations between COVID-19 hospitalization and inhalation substance use phenotypes. Cannabis Use Disorder (CUD) is highlighted in green. CPD = Cigarettes per day, Age Smoke $=$ Age of first becoming a regular smoker, Ever Smoke = Ever being a regular smoker, Cannabis Use = Any lifetime cannabis use. (B) Heat map of genetic correlations between all phenotypes included in the study. COVID-19 Hosp = COVID-19 hospitalization (Variable COVIDB2 from the COVID Host Genomics Consortium(4)). TDI = Townsend Deprivation Index, COVID-19 Hosp. = COVID-19 hospitalization, Edu Attain = Education 
medRxiv preprint doi: https://doi.org/10.1101/2020.11.15.20229971; this version posted November 18, 2020. The copyright holder for this preprint (which was not certified by peer review) is the author/funder, who has granted medRxiv a license to display the preprint in perpetuity.

It is made available under a CC-BY-NC-ND 4.0 International license .

attainment, BMI = Body Mass Index. FEV1 = Forced Expiration Volume for 1 second, Fast Gluc $=$ Fasting

Glucose. 
Table 1. COVID-19 Genetic Correlations When Accounting for Potential Confounding Variables

\begin{tabular}{|c|c|c|c|c|c|c|c|}
\hline Smoking phenotypes & $\beta$ CUD & $\beta$ Smoking phenotype ${ }^{\#}$ & $\beta \mathrm{EA}$ & $\beta$ TDI & $\beta \mathrm{BMI}$ & $\beta$ FEV & $\beta$ Fast Glu \\
\hline Cannabis Use & $0.566^{\star}$ & -0.362 & -0.004 & -0.109 & $0.288^{*}$ & 0.093 & -0.001 \\
\hline CPD & $0.358^{\star}$ & 0.044 & $-0.253^{\star}$ & $-0.256^{\star}$ & $0.292^{\star}$ & 0.075 & 0.032 \\
\hline Age Smoke & $0.339^{\star}$ & -0.090 & $-0.220^{\star}$ & -0.265 & $0.296^{*}$ & 0.068 & 0.034 \\
\hline Smoking Cessation & $0.379^{\star}$ & $-0.345^{\star}$ & -0.140 & $-0.375^{\star}$ & $0.288^{*}$ & 0.083 & 0.026 \\
\hline Ever Smoke & $0.407^{*}$ & -0.091 & $-0.260^{\star}$ & -0.221 & $0.311^{*}$ & 0.075 & 0.026 \\
\hline
\end{tabular}

Note. Standardized beta estimates for Cannabis Use Disorder (CUD) and smoking phenotypes were taken from a multiple regression parameterized in gSEM. "Smoking phenotypes were entered and tested separately to avoid multicollinearity amongst tobacco phenotypes. Each row represents genetic correlations with COVID-19 hospitalization from one model. The multiple rows indicate the separate models run substituting each tobacco phenotype (listed in the first column). The model was COVID hospitalization = CUD + Smoking Phenotype + BMI + Townsend Deprivation index + Educational Attainment + Forced Expiration + Fasting Glucose + error. The original GWAS accounted for standard GWAS covariates (age, sex, genetic principal components, etc). Bold ${ }^{*}$ represents $p<.05$. MJ Use = Marijuana Use, CPD $=$ Cigarettes per day, Age Smoke = Age started smoking regularly, Ever Smoke = Ever smoked regularly. 